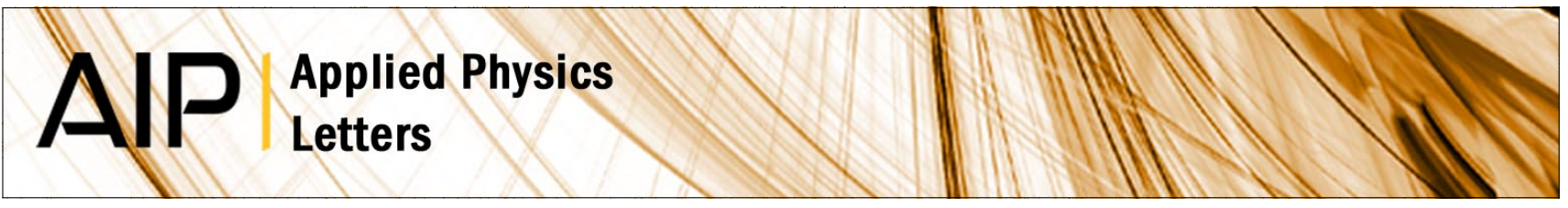

\title{
Electron-state engineering of bilayer graphene by ionic molecules
}

Nguyen Thanh Cuong, Minoru Otani, and Susumu Okada

Citation: Appl. Phys. Lett. 101, 233106 (2012); doi: 10.1063/1.4769098

View online: http://dx.doi.org/10.1063/1.4769098

View Table of Contents: http://apl.aip.org/resource/1/APPLAB/v101/i23

Published by the American Institute of Physics.

\section{Related Articles}

Diamond as an inert substrate of graphene

J. Chem. Phys. 138, 054701 (2013)

Barrier tunneling time of an electron in graphene

J. Appl. Phys. 113, 043714 (2013)

Tunable bandgap of a single layer graphene doped by the manganese oxide using the electrochemical doping Appl. Phys. Lett. 102, 032106 (2013)

Metal-semiconductor-metal photodetectors based on graphene/p-type silicon Schottky junctions Appl. Phys. Lett. 102, 013110 (2013)

Anomalous length dependence of the conductance of graphene nanoribbons with zigzag edges J. Chem. Phys. 138, 014704 (2013)

\section{Additional information on Appl. Phys. Lett.}

Journal Homepage: http://apl.aip.org/

Journal Information: http://apl.aip.org/about/about_the_journal

Top downloads: http://apl.aip.org/features/most_downloaded

Information for Authors: http://apl.aip.org/authors

\section{ADVERTISEMENT} \section{AIP $\begin{gathered}\text { Applied Physics } \\ \text { Letters }\end{gathered}$}

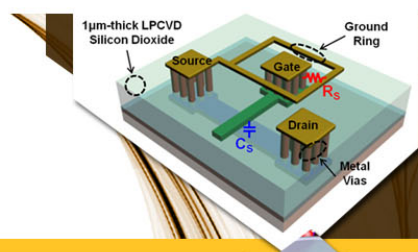

\section{SURFACES AND} INTERFACES

Focusing on physical, chemical, biological structural, optical, magnetic and electrical properties of surfaces and interfaces, and more..

\section{EXPLORE WHAT'S NEW IN APL}

SUBMIT YOUR PAPER NOW!
ENERCY CONVERSION AND STORACE 


\title{
Electron-state engineering of bilayer graphene by ionic molecules
}

\author{
Nguyen Thanh Cuong, ${ }^{1,2, a)}$ Minoru Otani, ${ }^{1,2}$ and Susumu Okada ${ }^{2,3}$ \\ ${ }^{1}$ Nanosystem Research Institute, National Institute of Advanced Industrial Science and Technology (AIST), \\ Tsukuba 305-8568, Japan \\ ${ }^{2}$ Japan Science and Technology Agency, CREST, 7 Gobancho, Chiyoda, Tokyo 102-0076, Japan \\ ${ }^{3}$ Graduate School of Pure and Applied Sciences, University of Tsukuba, 1-1-1 Tennodai, \\ Tsukuba 305-8571, Japan
}

(Received 25 September 2012; accepted 13 November 2012; published online 4 December 2012)

\begin{abstract}
Based on the first-principles total-energy calculations, we demonstrate the possibility of controlling the band-gap and carrier type of bilayer graphene using ionic molecules. Our calculations suggest that bilayer graphene sandwiched by a pair of cation-anion molecules is a semiconductor with a moderate energy gap of $0.26 \mathrm{eV}$ that is attributable to the strong local dipole field induced by the cation-anion pair. Furthermore, we can control the semiconducting carrier type-intrinsic, $p$-type, or $n$-type - of bilayer graphene sandwiched by ionic molecules by changing the cation-anion pair. (C) 2012 American Institute of Physics. [http://dx.doi.org/10.1063/1.4769098]
\end{abstract}

Graphene has stimulated intense interest not only in the field of the low-dimensional sciences but also in the electronic device engineering because of its unique structural and electronic properties. ${ }^{1-3}$ Graphene has a pair of linear dispersion bands at the Fermi level that confer a remarkable carrier mobility of up to $200000 \mathrm{~cm}^{2} /$ Vs. However, due to the absence of a band-gap, graphene shows an intrinsic leakage current in the off state, which limits its usage in logical circuits devices. ${ }^{4}$ Therefore, many studies are focusing on developing methods to control the electronic structure of graphene. In particular, the band gap engineering has attracted much attention for realizing the graphene-based semiconductor electronics devices. Indeed, many theoretical and experimental studies have designed and synthesized several derivatives of graphene having a large band-gap, such as graphene nanoribbon, ${ }^{5,6}$ graphene nanomesh, ${ }^{7}$ functionalized graphene, ${ }^{8}$ bilayer graphene under an external electric field,,$^{9-11}$ or molecular-doped bilayer graphene. ${ }^{12-15}$ However, these methods have several disadvantages related to cost, performance, accessibility, and controllability. Therefore, there continues to exist a need to develop a simple and less expensive method for synthesizing the semiconducting graphene.

Recently, ionic liquids have been used to the exfoliation of few-layer graphene sheets of high concentration and large area. ${ }^{16,17}$ An ionic liquid is an organic salt of high ionic conductivity and low melting point (below $100{ }^{\circ} \mathrm{C}$ ), and it consists of cation and anion ionic molecules. Hybrid materials comprising graphene and ionic molecules are expected to exhibit the unique properties that are not possessed by each constituent alone, and that should be suitable for application in various types of devices. ${ }^{18,19}$ At the same time, the weak interaction between graphene and the ionic molecules essentially preserves the fundamental electronic properties of each constituent. ${ }^{20}$ In this work, we propose a simple procedure for synthesizing semiconducting graphene, and for controlling its conducting properties using cation and anion ionic molecules adsorbing to two surfaces of graphene. Our first-principles calculations show that the bilayer graphene

a)Electronic mail: cuong-nguyen@aist.go.jp. sandwiched by the anion and cation molecules is a semiconductor with $0.26 \mathrm{eV}$ band-gap due to the strong local dipole moment induced by the cation-anion pair. Furthermore, we can control the type of semiconducting carrier of the sandwiched bilayer graphene-intrinsic, $p$-type, or $n$-type-by changing the cation-anion pair.

All the theoretical calculations have been performed based on the density functional theory ${ }^{21,22}$ as implemented in the STATE code. ${ }^{23}$ We use the local density approximation (LDA) to express the exchange-correlation energy, ${ }^{24,25}$ because the LDA well reproduces the experimental interlayer distance between graphene layers. The valence wave functions and augmented charge density are expanded in terms of a plane-wave basis set with cutoff energies of $25 \mathrm{Ry}$ and $225 \mathrm{Ry}$, respectively. The ultrasoft pseudopotentials are adopted to describe the electron-ion interaction. ${ }^{26}$ The Brillouin-zone integration is performed with the $\Gamma$-centered $6 \times 6 \times 1$ uniform $k$-mesh for self-consistent electronic structure calculations. All atoms are fully optimized until the remaining force acting on an atom is less than $0.005 \mathrm{Ry} / \AA$. The general direct inversion in the iterative subspace minimization scheme is used for the geometry optimization.

We considered a slab model in which AB-stacking bilayer graphene is sandwiched by three different pairs of cation-anion molecules: (i) tetrafluoroborate $\left(\mathrm{BF}_{4}^{-}\right)$anion/ 1-ethyl-1-methyl pyrrolidinum cation (S1 structure), (ii) tetrafluoroborate $\left(\mathrm{BF}_{4}^{-}\right)$anion/1-ethyl-pyridinium cation (S2 structure), and (iii) methylcarbonate $\left(\mathrm{CH}_{3} \mathrm{CO}_{3}^{-}\right)$anion/ 1-ethyl-3-methyl imidazolium cation ( $\mathrm{S} 3$ structure), respectively, as shown in Fig. 1. To simulate the dilute coverage of ionic molecules, we imposed the $6 \times 6$ lateral periodicity of bilayer graphene. To avoid the spurious electrostatic interactions with the periodic images in the slab calculation, we adopted the effective screen medium method ${ }^{27,28}$ with a vacuum region of $30 \AA$.

Figure 2(a) shows the electronic energy band of the bilayer graphene sandwiched by tetrafluoroborate $\left(\mathrm{BF}_{4}^{-}\right)$ anion and 1-ethyl-3-methyl pryrolidium cation (S1 structure). We find that this hybrid system is a direct-gap semiconductor with a moderate energy gap of $0.26 \mathrm{eV}$ between 


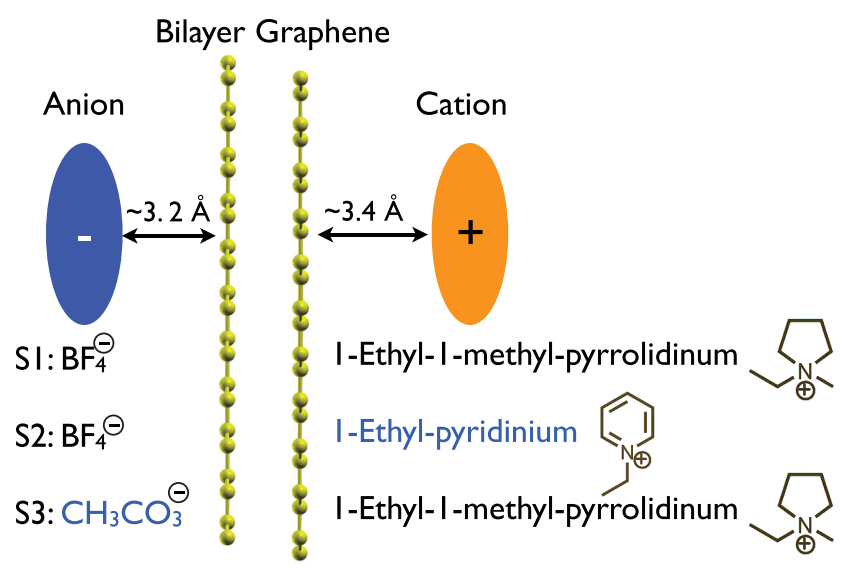

FIG. 1. Structural models of bilayer graphene sandwiched by ionic molecules: (S1) tetrafluoroborate $\left(\mathrm{BF}_{4}^{-}\right)$anion/1-ethyl-1-methyl pyrrolidinum cation, (S2) tetrafluoroborate $\left(\mathrm{BF}_{4}^{-}\right)$anion/1-ethyl-pyridinium cation, and (S3) methylcarbonate $\left(\mathrm{CH}_{3} \mathrm{CO}_{3}^{-}\right)$anion/1-ethyl-3-methyl imidazolium cation.

the conduction and the valence bands near the $\Gamma$-point. ${ }^{29} \mathrm{It}$ should be noted that the $K$ and $K^{\prime}$ points (Dirac points) are folded into the $\Gamma$ point in the graphene with $6 \times 6$ lateral periodicity. The gap value is comparable to those obtained in bilayer graphene under extremely high external electric field ${ }^{9-11}$ or that with doped molecules. ${ }^{12-15}$ In this structure, the top of the valence band and the bottom of the conduction band possess their $\pi$-state nature and are localized on the bilayer graphene. Therefore, bilayer graphene sandwiched by this cation-anion pair exhibits the characteristic features of an intrinsic semiconductor. This fact highlights the great advantage of using ionic molecules in a sandwich structure as opposed to using other molecules adsorbed on bilayer graphene for realizing the semiconducting graphene. ${ }^{12-15}$ In these doping procedures, the states of the guest molecules usually lie in the gap of the bilayer graphene which could affect the performance of graphene-based devices. ${ }^{13}$

We explore the physical origin of the moderate band-gap emerging in bilayer graphene sandwiched by a cation-anion pair by investigating the electrostatic potential profile along the direction normal to the graphene layers. Figure 2(b) shows the averaged electrostatic potential along the $z$-axis which corresponds to the normal direction to the graphene layers.
We find that the electrostatic potential at the right cell boundary is lower by $\sim 5 \mathrm{eV}$ than that at the left cell boundary due to the work function difference between the anion and cation molecules adsorbed on the graphene surfaces. Therefore, the ionic molecules induce an effective electric field perpendicular to the layers of bilayer graphene. This electric field leads to an electrostatic potential (on-site energy) difference of $0.75 \mathrm{eV}$ between the right and the left layers of bilayer graphene. According to this potential energy difference, the sandwiched bilayer graphene has a finite energy gap around the Dirac point due to the band shift and repulsion between two pairs of linear dispersion bands, as in the case of bilayer graphene under an external electric field. ${ }^{9-11}$ Dividing the potential difference by the interlayer spacing, the effective electric field applied on bilayer graphene is found to be $2.24 \mathrm{~V} / \mathrm{nm}$. Therefore, the fundamental energy gap and electronic structure of bilayer graphene can be controlled by tuning the cation-anion pair that causes the characteristic strength of the local electric field across the sandwiched bilayer graphene. Note that the fundamental energy gap under the calculated field is almost the same as that of the pristine bilayer graphene under an external electric field of about $2.2 \mathrm{~V} / \mathrm{nm}$ induced by two planar electrodes. ${ }^{30}$ However, a more thorough investigation should reveal the effect of ionic adsorbates on the electronic structures of each layer of bilayer graphene as in the case of the graphene on substrates. ${ }^{31}$

For realizing the graphene-based logic devices and circuits, it is important to obtain not only an intrinsic semiconducting graphene but also both $n$-type and $p$-type semiconducting graphene. As discussed above, the semiconducting properties of bilayer graphene can be controlled by tuning the molecule species of cation-anion pair. Figure 3(a) shows the electronic energy band of the bilayer graphene sandwiched by tetrafluoroborate $\left(\mathrm{BF}_{4}^{-}\right)$anion, and 1-ethyl-pyridinium cation (S2 structure). As in the case of the S1 structure, we find that the valence and conduction bands of the sandwiched bilayer graphene no longer touch each other but have an energy gap of $0.28 \mathrm{eV}$ around the $\Gamma$-point. On the other hand, in sharp contrast to the S1 structure, the Fermi level shifts downward and is located near the top of the valence band of bilayer graphene. Thus, the lowest unoccupied (LU) state of 1-ethyl- (a)

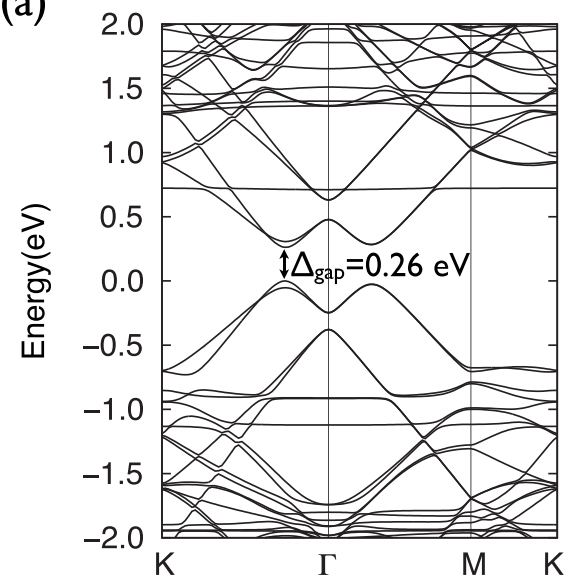

(b)

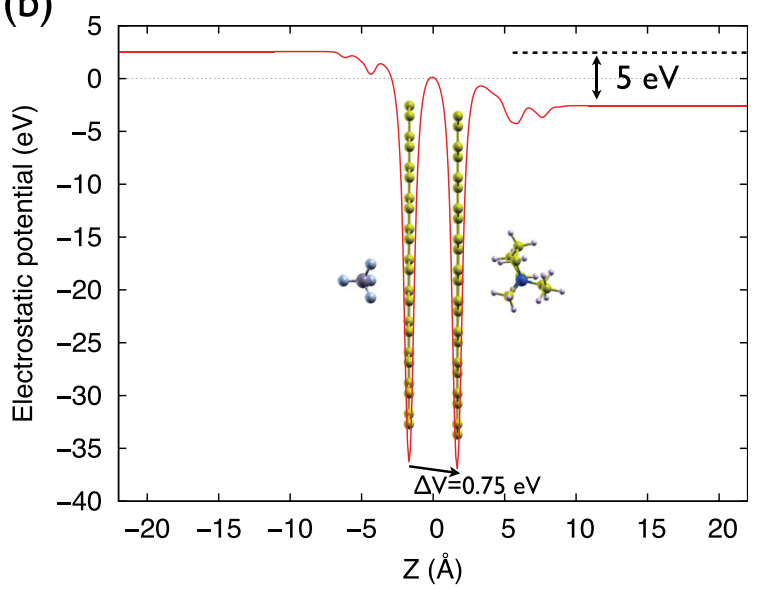

FIG. 2. (a) Electronic energy band of bilayer graphene sandwiched by tetrafluoroborate (BF $\left.{ }_{4}^{-}\right)$anion and 1-ethyl-1-methyl pyrrolidinum cation (S1 structure). Energies are measured from that of the Fermi level. (b) Average electrostatic potential profile along the $z$-axis normal to the graphene layers. 

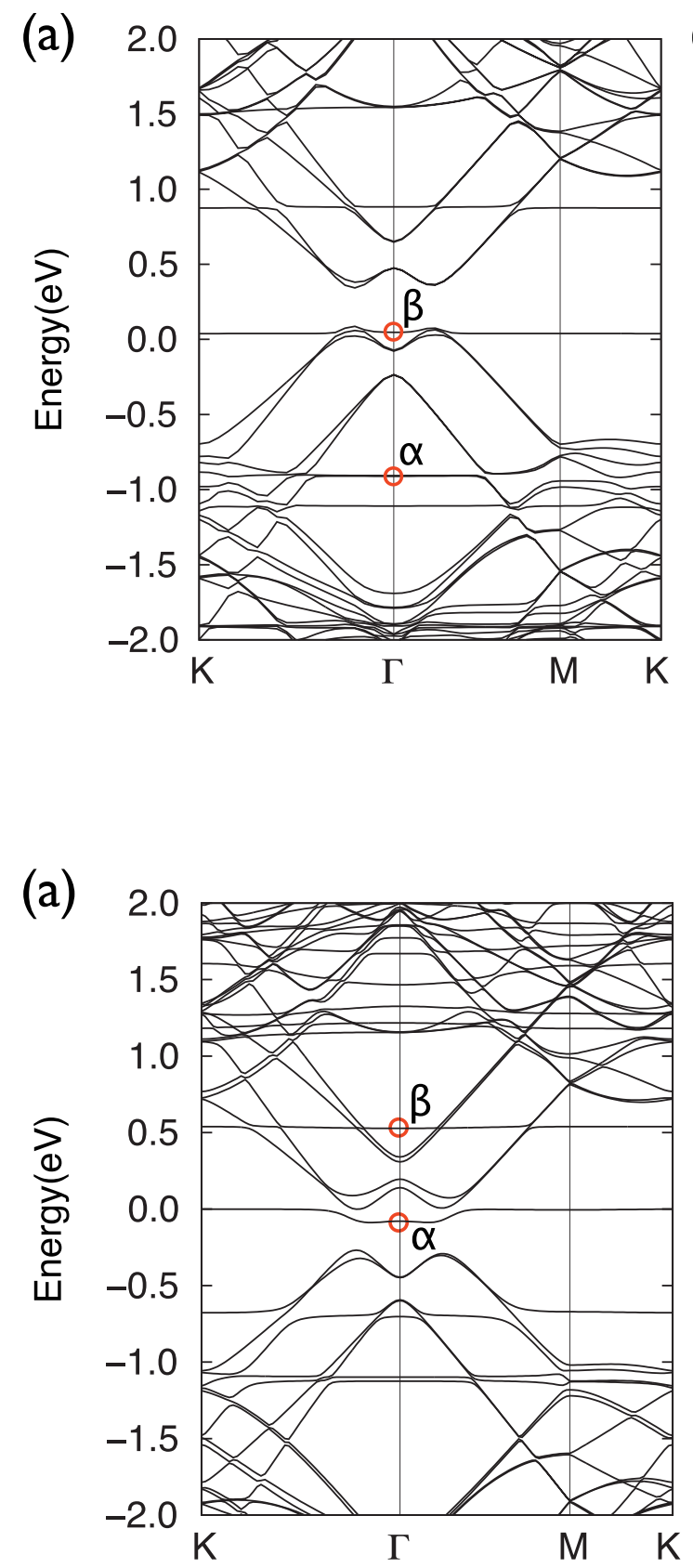
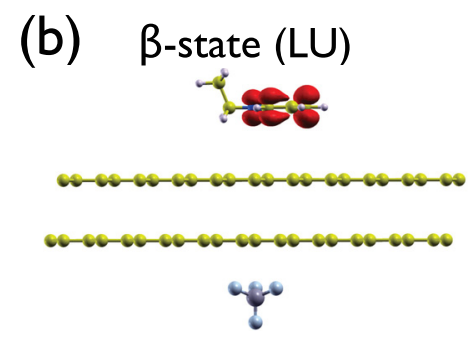

\section{$\alpha$-state (HO)}

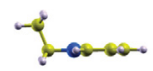

$20-60-60-60-60-60-60-00-60-60-60$

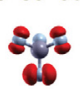

(b) $\beta$-state (LU)
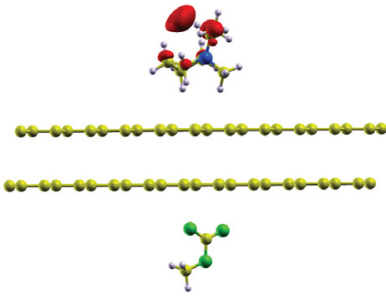

$\alpha$-state (HO)

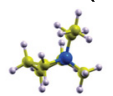

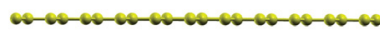

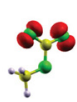

FIG. 3. (a) Electronic energy band of bilayer graphene sandwiched by tetrafluoroborate $\left(\mathrm{BF}_{4}^{-}\right)$anion and 1-ethyl-pyridinium cation (S2 structure). Energies are measured from that of the Fermi level. (b) Wavefunction distribution of LU and $\mathrm{HO}$ states of ionic molecules at the $\Gamma$-point.
FIG. 4. (a) Electronic energy band of bilayer graphene sandwiched by methylcarbonate $\left(\mathrm{CH}_{3} \mathrm{CO}_{3}^{-}\right)$anion and 1-ethyl-3-methyl imidazolium (S3 structure). Energies are measured from that of the Fermi level. (b) Wavefunction distribution of LU and $\mathrm{HO}$ states of ionic molecules at the $\Gamma$-point. (a)

$$
\text { P-type }
$$

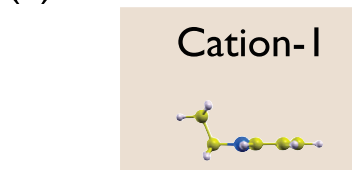

n-type

\section{Cation- II}

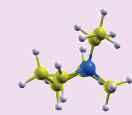

Bilayer Graphene

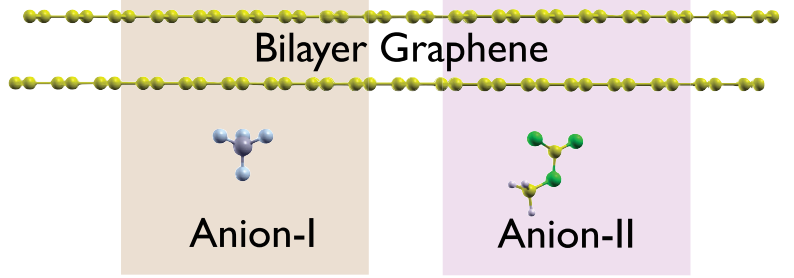

(b)

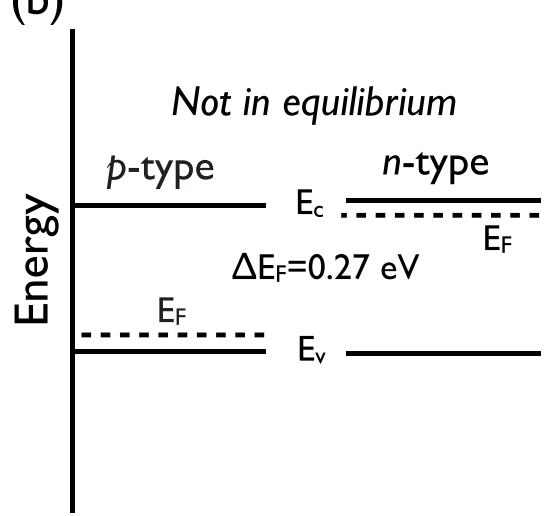

FIG. 5. (a) A structure model of all carbon $p$ - $n$ junction device, in which half of the upper/lower layers of graphene is covered by the tetrafluoroborate anion I/1-ethyl-pyridinium cation I, respectively, whereas the remaining area of the layers is covered by the methylcarbonate anion II/1-ethyl-3-methyl imidazolium cation II, respectively. (b) Diagram of energy bands of two separate $p$-type and $n$-type doping bilayer graphene semiconductors. 
pyridinium cation molecule $[\beta$-state in Fig. 3(b)] acts as a shallow level for the valence band of graphene, resulting in hole injection into the graphene layers. Therefore, this structure is one of the plausible candidates for $p$-type doping graphene semiconductors.

In addtion to the $p$-type doping graphene semiconductor, an $n$-type doping bilayer graphene semiconductor could be achieved by choosing an appropriate cation-anion pair. Figure 4(a) shows the electronic energy band of bilayer graphene sandwiched by methylcarbonate $\left(\mathrm{CH}_{3} \mathrm{CO}_{3}^{-}\right)$anion and 1-ethyl-3-methyl imidazolium cation ( $\mathrm{S} 3$ structure). This sandwiched bilayer graphene also possesses a gap of $0.32 \mathrm{eV}$ between the valence and the conduction bands around the $\Gamma$-point, in a manner similar to the bilayer graphene sandwiched by another cation-anion pair. In sharp contrast to the previous two structures, a filled and flat dispersion band emerges near the bottom of the conduction band of this bilayer graphene: this flat band possesses the character of the highest occupied (HO) state of a $\mathrm{CH}_{3} \mathrm{CO}_{3}^{-}$anion molecule [ $\alpha$-state in Fig. 4(b)]. Therefore, this state acts as a donor state for the conduction band of bilayer graphene that leads to an $n$-type doping semiconductor as in the case of the carbon nanotube encapsulating azafullerene $\mathrm{C}_{59} \mathrm{~N} .{ }^{32}$

By tuning the ionic molecule species, we can control the electronic structure of bilayer graphene to achieve the intrinsic, $p$-type, and $n$-type semiconducting bilayer graphene. This suggests that all carbon $p-n$ junctions could be designed by depositing two cation-anion pairs to the bilayer graphene. A plausible device structure is shown in Fig. 5(a): half of upper/lower layers of graphene is covered by the tetrafluoroborate anion/1-ethyl-pyridinium cation, respectively, whereas the remaining area of the layers is covered by the methylcarbonate anion/1-ethyl-3-methyl imidazolium cation, respectively. As shown in Fig. 5(b), in this case, an electric double layer is formed at the interface between the $p$-type and $n$-type areas of bilayer graphene due to the unbalancing in the Fermi level of $0.27 \mathrm{eV}$.

In summary, we have investigated the electronic properties of bilayer graphene sandwiched by ionic molecules based on first-principles total-energy calculations in the framework of the density functional theory. We find that bilayer graphene sandwiched by the cation and anion molecules is an intrinsic semiconductor with a direct energy gap of $0.26 \mathrm{eV}$. The work function difference of the anion and cation molecules adsorbed on the graphene surfaces induces a strong effective electric field of $2.2 \mathrm{~V} / \mathrm{nm}$ perpendicular to the graphene layers. This effective electric field leads to a difference in the on-site energy between graphene layers, resulting in a semiconducting character with a finite energy gap. Furthermore, the $p$-type or $n$-type doping bilayer graphene semiconductors can be achieved by choosing an appropriate cation-anion pair. This suggests that all carbon $p-n$ junction devices can be designed by depositing two cation-anion pairs to the bilayer graphene. Our calculations clearly demonstrate that graphene-based semiconducting devices can be realized using a hybrid structure comprising graphene and ionic molecules.

This work was partly supported by CREST, Japan Science and Technology Agency, and a Grant-in-Aid for scientific research from the Ministry of Education, Culture, Sports, Science and Technology of Japan.

${ }^{1}$ K. S. Novoselov, A. K. Geim, S. V. Morozov, D. Jiang, Y. Zhang, S. V. Dubonos, I. V. Grigorieva, and A. A. Firsov, Science 306, 666 (2004).

${ }^{2}$ X. Du, I. Skachko, A. Barker, and E. Y. Andrei, Nat. Nanotechnol. 3, 491 (2008).

${ }^{3}$ A. K. Geim and K. S. Novoselov, Nature Mater. 6, 183 (2007).

${ }^{4}$ F. Schwierz, Nat. Nanotechnol. 5, 487 (2010).

${ }^{5}$ M. Fujita, K. Wakabayashi, K. Nakada, and K. Kusukabe, J. Phys. Soc. Jpn. 65, 1920 (1996).

${ }^{6}$ M. Y. Han, B. Özyilmaz, Y. Zhang, and P. Kim, Phys. Rev. Lett. 98, 206805 (2007).

${ }^{7}$ J. Bai, X. Zhong, S. Jiang, Y. Huang, and X. Duan, Nat. Nanotechnol. 5, 190 (2010).

${ }^{8}$ J. T. Robinson, J. S. Burgess, C. E. Junkermeier, S. C. Badescu, T. L. Reinecke, F. K. Perkins, M. K. Zalalutdniov, J. W. Baldwin, J. C. Culbertson, P. E. Sheehan, and E. S. Snow, Nano Lett. 10, 3001 (2010).

${ }^{9}$ J. B. Oostinga, H. B. Heersche, X. Liu, A. F. Morpurgo, and L. M. K. Vandersypen, Nature Mater. 7, 151 (2008).

${ }^{10}$ Y. Zhang, T.-T. Tang, C. Girit, Z. Hao, M. C. Martin, A. Zettl, M. F. Crommie, Y. R. Shen, and F. Wang, Nature Mater. 459, 820 (2009).

${ }^{11}$ H. Miyazaki, K. Tsukagoshi, A. Kanda, M. Otani, and S. Okada, Nano Lett. 10, 3888 (2010).

${ }^{12}$ J. Park, S. B. Jo, Y.-J. Yu, Y. Kim, J. W. Yang, W. H. Lee, H. H. Kim, B. H. Hong, P. Kim, K. Cho, and K. S. Kim, Adv. Mater. 24, 407 (2012).

${ }^{13}$ D. L. Duong, S. M. Lee, S. H. Chae, Q. H. Ta, S. Y. Lee, G. H. Han, J. J. Bae, and Y. H. Lee, Phys. Rev. B 85, 205413 (2012).

${ }^{14}$ W. Zhang, C.-T. Lin, K.-K. Liu, T. Tite, C.-Y. Su, C.-H. Chang, Y.-H. Lee, C.-W. Chu, K.-H. Wei, J.-L. Kuo, and L.-J. Li, ACS Nano 5, 7517 (2011).

${ }^{15}$ X. Tian, J. Xu, and X. Wang, J. Phys. Chem. B 114, 11377 (2010).

${ }^{16}$ X. Wang, P. F. Fulvio, G. A. Baker, G. M. Veith, R. R. Unocic, S. M. Mahurin, M. Chi, and S. Dai, Chem. Commun. 46, 4487 (2010).

${ }^{17}$ D. Nuvoli, L. Valentini, V. Alzari, S. Scognamillo, S. B. Bon, M. Piccinini, J. Illescas, and A. Mariani, J. Mater. Chem. 21, 3428 (2011).

${ }^{18}$ Q. Ji, I. Honma, S.-M. Paek, M. Akada, J. P. Hill, A. Vinu, and K. Ariga, Angew. Chem., Int. Ed. 49, 9737 (2010).

${ }^{19}$ Y. Shim, Y. Jung, and H. J. Kim, J. Phys. Chem. C 115, 23574 (2011).

${ }^{20}$ M. H. Ghatee and F. Moosavi, J. Phys. Chem. C 115, 5626 (2011).

${ }^{21}$ P. Hohenberg and W. Kohn, Phys. Rev. 136, B864 (1964).

${ }^{22}$ W. Kohn and L. J. Sham, Phys. Rev. 140, A1133 (1965).

${ }^{23}$ Y. Morikawa, H. Ishii, and K. Seki, Phys. Rev. B 69, 041403(R) (2004).

${ }^{24}$ D. M. Ceperley and B. J. Alder, Phys. Rev. Lett. 45, 566 (1980).

${ }^{25}$ J. P. Perdew and A. Zunger, Phys. Rev. B 23, 5048 (1981).

${ }^{26}$ D. Vanderbilt, Phys. Rev. B 41, 7892 (1990).

${ }^{27}$ M. Otani and O. Sugino, Phys. Rev. B 73, 115407 (2006).

${ }^{28}$ I. Hamada, M. Otani, O. Sugino, and Y. Morikawa, Phys. Rev. B 80, 165411 (2009).

${ }^{29}$ Note that the density functional theory calculations usually underestimate the band-gap energy of semiconductors.

${ }^{30}$ M. Otani and S. Okada, J. Phys. Soc. Jpn. 79, 073701 (2010).

${ }^{31}$ N. T. Cuong, M. Otani, and S. Okada, Phys. Rev. Lett. 106, 106801 (2011).

${ }^{32}$ N. T. Cuong, M. Otani, Y. Iizumi, T. Okazaki, G. Rotas, N. Tagmatarchis, Y. Li, T. Kaneko, R. Hatakeyama, and S. Okada, Appl. Phys. Lett. 99, 053105 (2011). 